\title{
Wilms tumor: 15 years of experience at a children's hospital in Córdoba, Argentina
}

\author{
Claudia Seminara, M.D. ${ }^{a}$, M. Celia Planells, M.D. ${ }^{a}$, Ramón E. Pogonza, M.D. ${ }^{b}$ and Miriam Morales, M.D. ${ }^{a}$ \\ Collaborators: Celina Castro, M.D. ${ }^{c}$, Ariel Sferco, M.D. ${ }^{d}$, Arturo Sentagne, M.D. ${ }^{d}$, Melina Bertello, M.D. ${ }^{e}$ and \\ Roger Tissera, M.D.f
}

\begin{abstract}
The objective of this study was to describe the epidemiology, clinical presentation, treatment and nephrology follow-up of children with Wilms tumor. Data from 46 patients were collected. The clinical presentation occurred at a young age $(<40$ months old), with initial symptoms of pain, abdominal mass, and fever. The prevalent histology type was mixed nephroblastoma. All patients received pre-surgery chemotherapy followed by, in most cases, unilateral nephrectomy. Patients with a high histological risk had a 7.2 relative risk of death $(75 \%$ confidence interval: 1.5-33.7) compared to the rest, and a 2.5 relative risk of recurrence (75\% confidence interval: 1.0-6.4). Disease-free survival at 5 years was $70 \%$. Once cancer treatment was completed, $80 \%$ of patients maintained a stage-I kidney function. The most important prognostic factor was histology. These patients required a long-term nephrology follow-up. Key words: Wilms tumor, chronic kidney disease, nephrectomy.

http: / / dx.doi.org/10.5546/ aap.2019.eng.263
\end{abstract}

To cite: Seminara C, Planells MC, Pogonza RE, Morales M. Wilms tumor: 15 years of experience at a children's hospital in Córdoba, Argentina. Arch Argent Pediatr 2019;117(4):263-266.

a. Department of Nephrology.

b. Intensive Care Unit of Hospital de Niños de la Santísima Trinidad, Córdoba, Argentina.

Collaborators:

c. Department of Hematology and Oncology.

d. Department of Surgery. Hospital de Niños de la Santísima Trinidad.

e. Nephrology Residency Program of Sanatorio Allende.

f. Pediatric Residency Program of Hospital de Niños de la Santísima Trinidad.

E-mail address:

Claudia Seminara, M.D.: claudiaseminara@hotmail.com

Funding: None.

Conflict of interest: None.

Received: 4-20-2018

Accepted: 1-9-2019

\section{INTRODUCTION}

Wilms tumor is the most common pediatric kidney tumor and accounts for $6 \%$ of cancer in children. It has an incidence of 7.1 per million children aged 0-14 years. Thomas Rance first described this tumor in 1814. In 1899, German surgeon Max Wilms characterized it as a mixed renal tumor with the presence of three cell elements (blastema, epithelium, and stroma). ${ }^{1}$

Cases are sporadic, although 1-2\% corresponds to familial cases. Approximately $5-7 \%$ are bilateral tumors, and they may be synchronous (simultaneous) or metachronous (consecutive). ${ }^{2-4}$

Wilms tumor is related to mutations in the 11 p13 region (where the WT1 gene resides), at the long arm of chromosome 16 and chromosome 1. Some Wilms tumors are associated with genetic disorders, such as WAGR syndrome (Wilms tumor, aniridia, genitourinary anomalies, and mental retardation), Denys-Drash syndrome, and others. $^{4}$

The International Society of Pediatric Oncology (SIOP) has proposed a classification based on risk and histological type: low risk (necrotic, blastomatosis), intermediate (epithelial, stromal, mixed, and regressive), and high (blastemal, clear cell, and rhabdoid). ${ }^{5}$

The treatment recommended by the SIOP includes pre-surgery chemotherapy to reduce the tumor mass and thus simplify surgery, reduce the risk for tumor rupture and, therefore, the possibility of recurrence, and improve prognosis. ${ }^{3,6}$

Patients with Wilms tumor have a greater survival than those with other neoplasms.,

Wilms tumor staging criteria were defined by the National Wilms Tumor Study-4 (NWTS-4) (Table 1).

These patients require nephrology follow-up for an indefinite period due to their reduced renal mass, to detect toxicities caused by treatment (chemotherapy and radiation therapy) and any recurrent tumors. The risk for a second event varies from $5 \%$ to $7 \%$ at 30 years. 
The objective of this study was to describe the epidemiology, clinical presentation, treatment, and nephrology follow-up of children with Wilms tumor at a children's hospital in Córdoba between January 2000 and December 2015.

\section{POPULATION AND METHODS}

This was a retrospective, observational study based on the clinical records of patients with Wilms tumor seen at Hospital de Niños de La Santísima Trinidad between 2000 and 2015. Male and female patients aged 1 month to 15 years old diagnosed with unilateral or bilateral Wilms tumor and treated as per the SIOP guidelines were included. ${ }^{6}$

The outcome measures were age at diagnosis, sex, symptoms at onset, association with genetic disorders, unilateral or bilateral involvement, histological type as per the SIOP criteria, NWTS-4 staging criteria (Table 1), chemotherapy, radiation therapy, surgical treatment (total or partial nephrectomy or tumor resection), time between diagnosis and surgery, course (recurrence and/ or metastasis), arterial hypertension (blood pressure $>95^{\text {th }}$ percentile for sex, age, and height), ${ }^{8}$ proteinuria, kidney ultrasound (hypertrophy and residual renal mass alterations/relapse), stage of renal involvement, and mortality.

The renal involvement stage was established as per the Kidney Disease Improving Global Outcomes (KDIGO) 2012 guidelines, which are based on glomerular filtration rate as per Schwartz formula ( $\mathrm{k} x$ height/ serum creatinine $=\mathrm{mL} / \mathrm{min} / 1.73 \mathrm{~m}^{2}$ ). A glomerular filtration rate of above $90 \mathrm{~mL} / \mathrm{min} / 1.73 \mathrm{~m}^{2}$ was considered normal. ${ }^{9}$ Nephrology and laboratory controls were done once chemotherapy was completed.
Patients were assessed 1 and 5 years after Wilms tumor diagnosis.

Categorical outcome measures were compared using the $\chi^{2}$ test or Fisher's exact test, as applicable; a $p$ value $<0.05$ was considered significant. Continuous outcome measures were described as average, standard deviation, and lower and upper quartiles. The Epi Info 7 software was used.

The study was approved by the hospital's scientific committee.

\section{RESULTS}

During the study period, data from 46 patients diagnosed with Wilms tumor were collected. Their average age was 43 months at the time of diagnosis, with a lower and upper quartile of 17 and 39 months, respectively. Standard deviation: 28.2 months. In total, there were 24 female patients. In relation to their place of origin, 12/ 46 were from the capital city of Córdoba; 23 from other parts of the province of Córdoba; and 11 from other provinces.

The most common initial symptoms were abdominal swelling in 26 patients, abdominal pain in 9, fever in 8, and hematuria in 7. WAGR syndrome was found only in 3 patients, whereas 1 patient had idiopathic cardiomyopathy prior to diagnosis.

In relation to location, the tumor was in the right kidney in 25 patients; in the left kidney, in 19; and in both, in 2. Diagnosis was made clinically, with the help of imaging tests (ultrasound and computed tomography), and, in the case of bilateral involvement, with a contralateral biopsy.

TABle 1. National Wilms Tumor Study-4 staging criteria

Stage $\quad$ Description
I
Tumor is limited to the kidney and completely excised. The renal capsule is intact. There is no residual tumor
II
Tumor extends beyond the kidney but is completely excised. There is regional extension of the tumor (external
surface of the renal capsule into perirenal tissues). Blood vessels outside the kidney substance are infiltrated.
Blood vessels inside the kidney substance are infiltrated. Post-operative spillage confined to the flank.
- $\quad$ Lesidual tumor confined to abdomen (non-hematogenous spillage). Any of the following criteria may be observed:
$\quad$ Diffuse peritoneal contamination by the tumor; or implants are found on the peritoneal surfaces.
- Tumor extends beyond the surgical margins either microscopically or grossly.
HV $\quad$ Tumatogenous metastases are present (lung, liver, brain or bone).
B


According to histological risk, 5 patients had a low risk; 31, an intermediate risk; and 10, a high risk (Table 2). In relation to the NWTS-4 staging at the time of diagnosis, 23 patients corresponded to stage I; 5 , to stage II; 13 , to stage III; 4 , to stage IV; and 1 , to stage $\mathrm{V}$.

All patients received chemotherapy before the surgery, which consisted in a combination of vincristine, actinomycin, doxorubicin, etoposide, carboplatin, and ifosfamide. Only 11 patients received radiation therapy: 8 had an intermediate histological risk and 3, a high risk.

Surgical treatment consisted in total nephrectomy in 39 patients, partial nephrectomy in 2, unilateral nephrectomy plus contralateral tumor resection in 3 , tumor resection in 1 , and bilateral heminephrectomy in 1 .

During follow-up at 5 years, among the 46 patients, 15 had recurrence and/or metastasis (2 low-risk patients, 8 intermediate-risk patients, and 5 high-risk patients). Disease-free survival at 5 years was $70 \%$. Overall survival at 5 years was $87 \%$; it was $100 \%$ among those with a low histological risk, $96 \%$ among those with an intermediate risk, and $33 \%$ among those with a high risk.

Among the patients seen in our hospital, nephrology follow-up was not done in 5 because they were seen in other centers, and 6 patients died. Out of the 35 remaining patients, 5 developed proteinuria. Ultrasound controls showed that 19/35 patients had compensatory hypertrophy; 12 , a single kidney with a normal size; 2 , bilateral renal tumor; and 2, kidney malformation (horseshoe kidney).

Kidney function during nephrology follow-up (as per Schwartz formula) corresponded to stage I in 30 children, stage II in 4, and stage III in 1.

TABLE 2. Patient distribution by risk and tumor histological type, based on the International Society of Pediatric Oncology staging criteria

\begin{tabular}{llll}
\hline Risk & $\mathbf{N}$ & Histology & $\mathbf{N}$ \\
\hline \multirow{2}{*}{ Low risk } & 6 & Necrotic & 3 \\
& & Blastomatosis & 3 \\
Intermediate risk & 27 & Epithelial & 5 \\
& & Stromal & 5 \\
& & Mixed & 13 \\
& & Regressive & 4 \\
High risk & \multirow{2}{*}{13} & Blastemal & 9 \\
& & Clear cell & 3 \\
& & Rhabdoid & 1 \\
\hline
\end{tabular}

Patients with a high histological risk had a 7.2 relative risk (RR) of death $(75 \%$ confidence interval [CI]: 1.5-33.7) compared to intermediateand low-risk patients $(p=0.015)$; in addition, they had a $2.5 \mathrm{RR}$ of recurrence (75 \% CI: 1.0-6.4).

\section{DISCUSSION}

At the time of diagnosis of Wilms tumor, most patients were younger than 40 months. ${ }^{2}$ No sex prevalence and no significant difference in terms of the kidney laterality were observed.

Only 3 / 46 patients had a genetic disorder (WAGR syndrome in the 3 ), which was similar to the findings of previous studies. ${ }^{4}$

The most common clinical presentation was abdominal and lumbar pain, followed by abdominal mass, fever, and hematuria, also similar to what was observed in other studies. ${ }^{10,11}$

The prevalent histology type was mixed nephroblastoma. All patients received presurgery chemotherapy followed by, in most cases, unilateral total nephrectomy. The most important survival prognostic factor was histology as per the SIOP criteria; a high risk was associated with a greater mortality and recurrence.

Overall survival at 5 years of diagnosis in our patients was $87 \%$, which is slightly lower than what has been reported in other publications (more than $90 \%)^{12}$

No patient had end-stage renal disease, although follow-up duration was shorter in our patients compared to other sites, so it was not possible to make an adequate comparison. ${ }^{13}$

A glomerular filtration rate below $90 \mathrm{~mL} /$ $\mathrm{min} / 1.73$ (stage II-III chronic kidney disease) was observed in 5 out of 35 patients. Only 8 patients had proteinuria, defined as an early marker of chronic kidney disease caused by nephrotoxicity, chemotherapy, hyperfiltration, and radiation therapy. ${ }^{14}$ They did not receive follow-up with the department of nephrology because they were not referred to this area.

The continuous advances in knowledge about the genetic and molecular bases of Wilms tumor will help to establish future therapies customized to each patient's risk, as well as to identify new treatment strategies with an effectiveness and toxicity profile similar to that of standard chemotherapy. ${ }^{15}$

\section{CONCLUSION}

In our patients, Wilms tumor presented mostly in children younger than 40 months old, and no sex prevalence was observed. The most 
common initial symptoms were abdominal pain, abdominal mass, fever, and hematuria. Diseasefree survival at 5 years was $70 \%$.

Approximately $80 \%$ of patients maintained a stage-I kidney function once cancer treatment was completed. These patients require a long-term follow-up with the department of nephrology due to their mid- and long-term risk for complications secondary to the underlying disease and treatment.

\section{REFERENCES}

1. Ries LAG, Melbert D, Krapcho M, Mariotto A, et al (eds.). SEER Cancer Statistics Review 1975-2004. Bethesda: National Cancer Institute; 2007. [Accessed on: January $\left.11^{\text {th }}, 2019\right]$. Available at: https: / / seer.cancer.gov / archive / csr/1975_2004/.

2. Mambié Meléndez M, Guibelade del Castillo M, Nieto del Rincón N, Rodrigo Jiménez D, et al. Tumor de Wilms bilateral Metacrónico. An Pediatric (Barc). 2002; 56(3):24750 .

3. Mullen E, Weldon C, Kreidberg J. Pediatric Renal Tumors. In: Avner E, Harmon W, Niaudet P, Yoshikawa N (eds.). Pediatric Nephrology. $6^{\text {th }}$ ed. Heidelberg: Springer-Verlag; 2009.P.1431-55.

4. López L, Adragna M, Di Pinto D, Cacciavillano W, et al. Compromiso renal de los pacientes con Tumor de Wilms Bilateral. MedInfant. 2016; 23:74-8.

5. Vujanić GM, Sandstedt B, Harms D, Kelsey A, et al. Revised International Society of Paediatric Oncology (SIOP) working classification of renal tumors of childhood. Med Pediatr Oncol. 2002; 38(2):79-82.
6. Romao R, Lorenzo A. Renal function in patients with Wilms Tumor. Urol Oncol. 2016; 34(1):33-41.

7. KasteSC, DomeJS, Babyn PS, Graf NM, etal. Wilmstumour: prognostic factors, staging, therapy and late effects. Pediatr Radiol. 2008; 38(1):2-17.

8. National High Blood Pressure Education Program Working Group on High Blood Pressure in Children and Adolescent. The fourth report on the diagnosis, evaluation, and treatment of high blood pressure in children and adolescents. Pediatrics. 2004;114(2Suppl4th Report):555-76.

9. Stefanowicz J, Kosiak M, Romanowicz G, Owczuk R, et al. Glomerular filtration rate and prevalence of chronic kidney disease in Wilms' tumour survivors. Pediatr Nephrol. 2011; 26(5):759-66.

10. Illadea L, Hernandez-Marques C, Cormenzana M, Lassaletta A, et al. Tumor de Wilms: revisión de nuestra experiencia en los últimos 15 años. An Pediatr (Barc). 2018; 88(3):140-9.

11. Levitt G. Renal Tumours: Long-term outocome. Pediatr Nephrol. 2012; 27(6): 911-6.

12. Isa ON, Reyes CM, Russo NM. Resultado del tratamiento del tumor de Wilms en población Pediátrica. Rev Chil Pediatr. 2013; 84(6):628-34.

13. Kazama T, Nio M, Sasaki H, Fukuzawa T, Sato T. Estimated glomerular filtration rate after nephrectomy for Wilms tumor. Pediatr Int. 2018; 60(10):962-5.

14. Skinner R. Late renal toxicity of treatment for childhood malignancy: risk factors, long-term outcomes, and surveillance. Pediatr Nephrol. 2018; 33(2):215-25.

15. Dome JS, Graf N, Geller J, Fernandez C, et al. Advances in Wilms tumor treatment and biology: Progress Through internacional collaboration. J Clin Oncol. 2015; 33(27):29993007. 